\title{
NONLOCAL PROBLEMS FOR QUASILINEAR FUNCTIONAL PARTIAL DIFFERENTIAL EQUATIONS OF FIRST ORDER
}

\author{
JAN TURO
}
Abstract
Existence and uniqueness of almost everywhere solutions of nonlo- cal problems to functional partial differential systems in diagonal form are investigated. The proof is based on the characteristics and fixed point methods.

\section{Introduction}

For any metric spaces $X$ and $Y$ we denote by $C(X, Y)$ the set of all continuous functions from $X$ to $Y$. Let $a_{0}>0$ be a given constant and $I_{a_{0}}=\left[0, a_{0}\right] \times \mathbb{R}^{m}$. Write $D=[-\tau, 0] \times[-b, b]$, where $\tau \in$ $\mathbb{R}_{+}=[0,+\infty)$ and $b=\left(b_{1}, \ldots, b_{m}\right) \in \mathbb{R}_{+}^{m}$. For $z:\left[-\tau, a_{0}\right] \times \mathbb{R}^{m} \rightarrow \mathbb{R}^{n}$ and $(x, y)=\left(x, y_{1}, \ldots, y_{m}\right) \in I_{a_{0}}$, we define $z_{(x, y)}: D \rightarrow \mathbb{R}^{n}$ by $z_{(x, y)}(s, t)=z(x+s, y+t),(s, t) \in D$. Thus, we see that $z_{(x, y)}$ is a restriction of $z$ to the rectangle $[x-\tau, x] \times[y-b, y+b]$. Put $\Omega=\left[0, a_{0}\right] \times \mathbb{R}^{m} \times C\left(D, \mathbb{R}^{m}\right)$ and $I=[-\tau, 0] \times \mathbb{R}^{m}$.

We assume that $\varrho=\left[\varrho_{i j}\right]: \Omega \rightarrow \mathbb{R}^{n m}, i=1, \ldots, n, j=1, \ldots, m$, $f=\left(f_{1}, \ldots, f_{n}\right): \Omega \rightarrow \mathbb{R}^{n}, h_{k}=\left[h_{k i j}\right]: I \rightarrow \mathbb{R}^{n n}, i, j=1, \ldots, n$, $k=1, \ldots, r, \varphi=\left(\varphi_{1}, \ldots, \varphi_{n}\right): I \rightarrow \mathbb{R}^{n}$ are given functions.

We consider quasilinear hyperbolic systems of functional partial differential equations

(1) $D_{x} z_{i}(x, y)+\sum_{j=1}^{m} \varrho_{i j}\left(x, y, z_{(x, y)}\right) D_{y_{j}} z_{i}(x, y)=f_{i}\left(x, y, z_{(x, y)}\right)$,

$i=1, \ldots, n,(x, y) \in I_{a_{0}}$, with nonlocal condition

$$
z_{(0, y)}+\sum_{k=1}^{r}\left(h_{k}\right)_{(0, y)} z_{\left(a_{k}, y\right)}=\varphi_{(0, y)}, \quad y \in \mathbb{R}^{m}
$$


where $a_{k}, k=1, \ldots, r$, are finite numbers such that $0<a_{1}<a_{2}<\cdots<$ $a_{r} \leq a_{0}$.

The nonlocal condition (2) may be also written in the form

$$
z(x, y)+\sum_{k=1}^{r} h_{k}(x, y) z\left(a_{k}+x, y\right)=\varphi(x, y), \quad(x, y) \in I
$$

For $r=n, \tau=0$ and $h_{k i j}=h_{i j} \delta_{k i}\left(\delta_{k i}\right.$ is the Kronecker symbol) nonlocal boundary condition (2) reduces to the nonlocal condition "á la Cesari" [8], [1]. If $h_{k i j}=\delta_{k i} \delta_{i j}$ then (2) reduces to the Nicoletti condition [10], [12]. Furthermore, if all $a_{k}=0, k=1, \ldots, r$ then we get the usual Cauchy condition.

Nonlocal condition was considered for parabolic problems in [4], [5], $[\mathbf{8}]$, and for hyperbolic problems in $[\mathbf{2}],[\mathbf{3}],[\mathbf{6}],[\mathbf{9}]$. Mixed problems for system (1) in two independent variables were investigated in [10].

System (1) contains as particular cases the system of differential equations with a retarded argument, differential-integral systems and differential-functional equations with operators of the Volterra type (see Section 4).

In this paper, we consider the local existence and uniqueness of generalized solutions of nonlocal problem (1), (2). The method used in the paper is based on characteristics theory and the fixed point theorem.

\section{Assumptions and Lemma}

For $\eta=\left(\eta_{1}, \ldots, \eta_{k}\right) \in \mathbb{R}^{k}$ we write $|\eta|_{k}=\max \left\{\left|\eta_{i}\right|: 1 \leq i \leq k\right\}$. For the matrix $U=\left[u_{i j}\right], i=1, \ldots, n, j=1, \ldots, m$, we define $\|U\|=$ $\max \left\{\sum_{j=1}^{m}\left|u_{i j}\right|: 1 \leq i \leq n\right\}$. Let $\|v\|$ denote the supremum norm of $v \in C\left(D, \mathbb{R}^{n}\right)$ and $C\left(D, \mathbb{R}^{n} ; p\right)=\left\{v \in C\left(D, \mathbb{R}^{n}\right):\|v\| \leq p\right\}, p \in \mathbb{R}_{+}$. Let $L([\alpha, \beta], \mathbb{R})$ be the set of all integrable functions $l:[\alpha, \beta] \rightarrow \mathbb{R}$. We denote by $C_{L}\left(D, \mathbb{R}^{n}\right)$ the class of all functions $v \in C\left(D, \mathbb{R}^{n}\right)$ satisfying the condition

(4) $\quad|v(s, t)-v(\bar{s}, \bar{t})|_{n} \leq\left|\int_{s}^{\bar{s}} \omega(\xi) d \xi\right|+q|t-\bar{t}|_{m}, \quad(s, t),(\bar{s}, \bar{t}) \in D$

where $\omega \in L\left([-\tau, 0], \mathbb{R}_{+}\right), q \in \mathbb{R}_{+}(\omega$ and $q$ depend on $v)$. For $v \in$ $C_{L}\left(D, \mathbb{R}^{n}\right)$ we define $\|v\|_{L}=\|v\|_{*}+\|v\|$, where

$$
\|v\|_{*}=\inf \left\{q+\int_{-\tau}^{0} \omega(\xi) d \xi: q \text { and } \omega \text { satisfy }(4)\right\} .
$$


Let $C_{L}\left(D, \mathbb{R}^{n} ; p\right)=\left\{v \in C_{L}\left(D, \mathbb{R}^{n}\right):\|v\|_{L} \leq p\right\}, p \in \mathbb{R}_{+}$. Denote by $\Lambda$ the set of all functions $\lambda:\left[0, a_{0}\right] \times \mathbb{R}_{+} \rightarrow \mathbb{R}_{+}$such that $\lambda(\cdot, t) \in$ $L\left(\left[0, a_{0}\right], \mathbb{R}_{+}\right)$for each $t \in \mathbb{R}_{+}$and $\lambda(s, \cdot)$ is nondecreasing on $\mathbb{R}_{+}$for almost every (a.e.) $s \in\left[0, a_{0}\right]$.

\section{Assumption $H_{1}$. Suppose that}

1. the matrix-valued function $\varrho(\cdot, y, v):\left[0, a_{0}\right] \rightarrow \mathbb{R}^{n m}$ is measurable for every $(y, v) \in \mathbb{R}^{m} \times C\left(D, \mathbb{R}^{n}\right)$ and $\varrho(x, \cdot): \mathbb{R}^{m} \times C\left(D, \mathbb{R}^{n}\right) \rightarrow$ $\mathbb{R}^{n m}$ is continuous for a.e. $x \in\left[0, a_{0}\right]$;

2. there exists $d \in \Lambda$ such that

$$
\|\varrho(x, y, v)\| \leq d(x, p)
$$

for all $(y, v) \in \mathbb{R}^{m} \times C\left(D, \mathbb{R}^{n} ; p\right)$, a.e. $x \in\left[0, a_{0}\right] ;$

3. there exists $l \in \Lambda$ such that

$$
\|\varrho(x, y, v)-\varrho(x, \bar{y}, \bar{v})\| \leq l(x, p)\left[|y-\bar{y}|_{m}+\|v-\bar{v}\|\right]
$$

for all $(y, v),(\bar{y}, \bar{v}) \in \mathbb{R}^{m} \times C_{L}\left(D, \mathbb{R}^{n} ; p\right)$ and a.e. $x \in\left[0, a_{0}\right]$.

\section{Assumption $\mathrm{H}_{2}$. Suppose that}

1. there exist constants $\bar{p} \in\left(0, \frac{1}{2}\right], \bar{q}_{0} \in \mathbb{R}_{+}$and a function $\bar{\omega}_{0} \in$ $L\left([-\tau, 0], \mathbb{R}_{+}\right)$such that

$$
\begin{aligned}
& \sum_{k=1}^{r}\left\|h_{k}(x, y)\right\| \leq \bar{p} \\
& \sum_{k=1}^{r}\left\|h_{k}(x, y)-h_{k}(\bar{x}, \bar{y})\right\| \leq\left|\int_{x}^{\bar{x}} \bar{\omega}_{0}(s) d s\right|+\bar{q}_{0}|y-\bar{y}|_{m}
\end{aligned}
$$

2. there exist constants $p_{0}, q_{0} \in \mathbb{R}_{+}$and a function $\omega_{0} \in L\left([-\tau, 0], \mathbb{R}_{+}\right)$ such that

$$
\begin{aligned}
& |\varphi(x, y)|_{n} \leq p_{0}, \\
& |\varphi(x, y)-\varphi(\bar{x}, \bar{y})|_{n} \leq\left|\int_{x}^{\bar{x}} \omega_{0}(s) d s\right|+q_{0}|y-\bar{y}|_{m}
\end{aligned}
$$

for all $(x, y),(\bar{x}, \bar{y}) \in I$. 
For $a \in\left(0, a_{0}\right]$ we denote by $C_{\varphi, a}[p, \omega, q]$ the set of all functions $v \in$ $C\left([-\tau, a] \times \mathbb{R}^{m}, \mathbb{R}^{n}\right)$ such that

$$
\begin{aligned}
& |z(x, y)|_{n} \leq p, \\
& |z(x, y)-z(\bar{x}, \bar{y})|_{n} \leq\left|\int_{x}^{\bar{x}} \omega(s) d s\right|+q|y-\bar{y}|_{m}
\end{aligned}
$$

for $(x, y),(\bar{x}, \bar{y}) \in I_{a}$ and $v$ satisfies condition (3) on $I$.

We consider a Carathéodory solution of (1), (2). More precisely, a function $z$ is called a Carathéodory solution of problem (1), (2) provided the following conditions hold:

(i) $z \in C_{\varphi, a}[p, \omega, q]$;

(ii) $z$ satisfies (1) almost everywhere in $I_{a}$ and (2) everywhere in $\mathbb{R}^{n}$.

For $z \in C_{\varphi, a}[p, \omega, q]$ we consider the following problem

$$
\eta^{\prime}(t)=\varrho_{i}\left(t, \eta(t), z_{(t, \eta(t))}\right), \quad \eta(x)=y, \quad i=1, \ldots, n .
$$

Note that (5) is an ordinary differential equation. If Assumption $H_{1}$ is satisfied then for every $z \in C_{\varphi, a}[p, \omega, q]$ the right hand side of (5) satisfies Carathéodory assumptions and the following Lipschitz condition

$$
\left|\varrho_{i}\left(t, \xi, z_{(t, \xi)}\right)-\varrho_{i}\left(t, \bar{\xi}, z_{(t, \bar{\xi})}\right)\right| \leq l\left(t, r_{a}\right)(1-q)|\xi-\bar{\xi}|_{m},
$$

holds, where $r_{a}=p+q+\int_{-\tau}^{a} \omega(s) d s$. Thus, the existence and uniqueness of the solution $g_{i}[z](\cdot ; x, y):[0, a] \rightarrow \mathbb{R}$ of (5) follows from classical theorems.

Lemma 1. If Assumption $H_{1}$ is satisfied and $z, \bar{z} \in C_{\varphi, a}[p, \omega, q]$ then

(6) $\quad\left|g_{i}[z](t ; x, y)-g_{i}[z](t ; \bar{x}, \bar{y})\right|_{m}$

$$
\leq \exp \left[(1+q) \int_{t}^{x} l\left(s, r_{a}\right) d s\right]\left[\left|\int_{x}^{\bar{x}} d(s, p) d s\right|+|y-\bar{y}|_{m}\right]
$$

$t \in[0, \min (x, \bar{x})], i=1, \ldots, n$, and

(7) $\left|g_{i}[z](t ; x, y)-g_{i}[\bar{z}](t ; x, y)\right|_{m}$

$$
\leq \int_{t}^{x} l\left(s, r_{a}\right) d s \exp \left[(1+q) \int_{t}^{x} l\left(s, r_{a}\right) d s\right]\|z-\bar{z}\|_{a}, \quad t \in[0, x],
$$


where $\|\cdot\|_{a}$ denotes the supremum norm in the space $C\left(I_{a}, \mathbb{R}^{n}\right)$.

Proof: We will consider the case where $x \leq \bar{x}$. We have, by Assumption $H_{1}$,

$$
\begin{aligned}
& \left|g_{i}[z](t ; x, y)-g_{i}[z](t ; \bar{x}, \bar{y})\right|_{m} \leq|y-\bar{y}|_{m}+\int_{x}^{\bar{x}} d(s, p) d s \\
& \quad+\left|\int_{x}^{t} l\left(s, r_{a}\right)(1+q)\right| g_{i}[z](s ; x, y)-\left.g_{i}[z](s ; \bar{x}, \bar{y})\right|_{m} d s \mid, \quad t \in[0, x] .
\end{aligned}
$$

Hence,by the above inequality and by Gronwall's inequality we get (6). We consider the case $x>\bar{x}$ analogously to the case $x \leq \bar{x}$.

It follows, from Assumption $H_{1}$, that

$$
\begin{gathered}
\left|g_{i}[z](t ; x, y)-g_{i}[\bar{z}](t ; x, y)\right|_{m} \leq \mid \int_{x}^{t} l\left(s, r_{a}\right)\left[\left|g_{i}[z](s ; x, y)-g_{i}[\bar{z}](s ; x, y)\right|_{m}\right. \\
\left.+|| z_{\left(s, g_{i}[z](s ; x, y)\right)}-\bar{z}_{\left(s, g_{i}[\bar{z}](s ; x, y)\right)}||\right] d s \mid, \quad t \in[0, x] .
\end{gathered}
$$

Since

$$
\begin{aligned}
\left\|z_{\left(s, g_{i}[z](s ; x, y)\right)}-\bar{z}_{\left(s, g_{i}[\bar{z}(s ; x, y))\right.}\right\| & \\
& \leq\|z-\bar{z}\|_{a}+q\left|g_{i}[z](s ; x, y)-g_{i}[\bar{z}](s ; x, y)\right|_{m},
\end{aligned}
$$

we get

$$
\begin{aligned}
\mid g_{i}[z](t ; x, y)- & \left.g_{i}[\bar{z}](t ; x, y)\right|_{m} \leq\left|\int_{x}^{t} l\left(s, r_{a}\right) d s\right|\|z-\bar{z}\|_{a} \\
& +\left|\int_{x}^{t} l\left(s, r_{a}\right)(1+q)\right| g_{i}[z](s ; x, y)-g_{i}[\bar{z}](s ; x, y)|d s| .
\end{aligned}
$$

Utilizing the Gronwall's inequality, we obtain (7). The proof of Lemma 1 is complete.

\section{Assumption $H_{3}$. Suppose that}

1. the vector-valued function $f(\cdot, y, v):\left[0, a_{0}\right] \rightarrow \mathbb{R}^{n}$ is measurable for every $(y, v) \in \mathbb{R}^{m} \times C\left(D, \mathbb{R}^{n}\right)$ and $f(x, \cdot): \mathbb{R}^{m} \times C\left(D, \mathbb{R}^{n}\right) \rightarrow$ $\mathbb{R}^{n}$ is continuous for a.e. $x \in\left[0, a_{0}\right]$;

2. there exists $d_{1} \in \Lambda$ such that

$$
|f(x, y, v)|_{n} \leq d_{1}(x, p)
$$

for all $(y, v) \in \mathbb{R}^{m} \times C\left(D, \mathbb{R}^{n} ; p\right)$, a.e. $x \in\left[0, a_{0}\right]$;

3. there exists $l_{1} \in \Lambda$ such that

$$
|f(x, y, v)-f(x, \bar{y}, \bar{v})| \mid \leq l_{1}(x, p)\left[|y-\bar{y}|_{m}+\| v-\bar{v}||\right]
$$

for all $(y, v),(\bar{y}, \bar{v}) \in \mathbb{R}^{m} \times C_{L}\left(D, \mathbb{R}^{n} ; p\right)$ and a.e. $x \in\left[0, a_{0}\right]$. 


\section{The main theorem}

Theorem 1. Suppose that Assumptions $H_{1}-H_{3}$ are satisfied. Then there exist $a \in\left(0, a_{0}\right], p, q \in \mathbb{R}_{+}$and $\omega \in L\left([-\tau, a], \mathbb{R}_{+}\right)$such that problem (1), (2) has a unique solution $z$ in the class $C_{\varphi, a}[p, \omega, q]$.

Proof: First, we take $a$ so small that

$$
\begin{gathered}
\int_{0}^{a} d_{1}(t, p) d t \leq 1, \quad K_{a}=\exp \left[L_{a}(1+q)\right] \leq 2, \\
L_{1 a}(1+q) \leq 1, \quad R_{a} L_{a} K_{a}+L_{1 a}<\frac{1}{2},
\end{gathered}
$$

where $L_{a}=\int_{0}^{a} l(t, p) d t, L_{1 a}=\int_{0}^{a} l_{1}(t, p) d t$ and $R_{a}=q_{0}+\bar{q}_{0} p+\bar{p} q+$ $L_{1 a}(1+q)$.

Let us choose constants $p, q$ with

$$
\begin{aligned}
& p \geq(1-\bar{p})\left(1+p_{0}\right), \\
& q \geq 2(1-2 \bar{p})^{-1}\left(q_{0}+\bar{q}_{0} p+1\right)
\end{aligned}
$$

and function

$$
\omega(t)=\max \left\{q d(t, p)+d_{1}(t, p),(1-\bar{p})^{-1}\left[\omega_{0}(t)+p \bar{\omega}(t)\right]\right\} .
$$

We define the following operator

$$
\begin{aligned}
(T z)_{i}(x, y)= & \varphi_{i}\left(0, g_{i}(0 ; x, y)\right)-\sum_{k=1}^{r} h_{k i}\left(0, g_{i}(0 ; x, y)\right) z_{i}\left(a_{k}, g_{i}(0 ; x, y)\right) \\
& +\int_{0}^{x} f_{i}\left(t, g_{i}(t ; x, y), z_{\left(t, g_{i}(t ; x, y)\right)}\right) d t, \quad(x, y) \in I_{a} \\
(T z)_{i}(x, y)= & \varphi_{i}(x, y)-\sum_{k=1}^{r} h_{k i}(x, y) z_{i}\left(a_{k}+x, y\right), \quad(x, y) \in I
\end{aligned}
$$

From Assumptions $H_{2}, H_{3}$ and inequalities (8), (9), we obtain

$$
\begin{aligned}
\left|(T z)_{i}(x, y)\right| \leq & \left|\varphi_{i}\left(0, g_{i}(0 ; x, y)\right)\right| \\
& +\left|\sum_{k=1}^{r} h_{k i}\left(0, g_{i}(0 ; x, y)\right) z_{i}\left(a_{k}, g_{i}(0 ; x, y)\right)\right| \\
& +\left|\int_{0}^{x} f_{i}\left(t, g_{i}(t ; x, y), z_{\left(t, g_{i}(t ; x, y)\right)}\right) d t\right| \\
\leq & p_{0}+\bar{p} p+\int_{0}^{a} d_{1}(t, p) d t \leq p, \quad(x, y) \in I_{a}
\end{aligned}
$$


and

$$
\begin{aligned}
\left|(T z)_{i}(x, y)\right| \leq\left|\varphi_{i}(x, y)\right|+\left|\sum_{k=1}^{r} h_{k i}(x, y) z_{i}\left(a_{k}+x, y\right)\right| \\
\leq p_{0}+\bar{p} p \leq p, \quad(x, y) \in I .
\end{aligned}
$$

For any $(x, y),(\bar{x}, \bar{y}) \in I_{a}$, from Assumptions $H_{1}-H_{3}$ and Lemma 1 , we get

$$
\begin{gathered}
\left|(T z)_{i}(x, y)-(T z)_{i}(\bar{x}, \bar{y})\right| \leq R_{a}\left|g_{i}(t ; x, y)-g_{i}(t ; \bar{x}, \bar{y})\right|+\left|\int_{x}^{\bar{x}} d_{1}(t, p) d t\right| \\
\leq R_{a} K_{a}\left|\int_{x}^{\bar{x}} d(t, p) d t\right|+\left|\int_{x}^{\bar{x}} d_{1}(t, p) d t\right|+R_{a} K_{a}|y-\bar{y}|_{m} .
\end{gathered}
$$

For $(x, y),(\bar{x}, \bar{y}) \in I$, we have

$$
\begin{aligned}
&\left|(T z)_{i}(x, y)-(T z)_{i}(\bar{x}, \bar{y})\right| \leq\left|\int_{x}^{\bar{x}} \omega_{0}(t) d t\right|+\bar{p}\left|\int_{x}^{\bar{x}} \omega(t) d t\right| \\
&+p\left|\int_{x}^{\bar{x}} \bar{\omega}_{0}(t) d t\right|+\left(q_{0}+\bar{p} q+p \bar{q}_{0}\right)|y-\bar{y}|_{m} .
\end{aligned}
$$

Thus, from (8)-(10), we obtain

$$
\left|(T z)_{i}(x, y)-(T z)_{i}(\bar{x}, \bar{y})\right| \leq\left|\int_{x}^{\bar{x}} \omega(t) d t\right|+q|y-\bar{y}|_{m}, \quad(x, y) \in I_{a} .
$$

We see that $T z \in C_{\varphi, a}[p, \omega, q]$.

Now, we prove that $T$ is a contraction. Indeed, for any $z, \bar{z} \in C_{\varphi, a}[p, \omega, q]$ we have

$$
\begin{aligned}
& \left|(T z)_{i}(x, y)-(T \bar{z})_{i}(x, y)\right| \leq\left|\varphi_{i}(0, g[z](0 ; x, y))-\varphi_{i}(0, g[\bar{z}](0 ; x, y))\right| \\
& +\left|\sum_{k=1}^{r}\left[h_{k i}\left(0, g_{i}[z](0 ; x, y)\right)-h_{k i}\left(0, g_{i}[\bar{z}](0 ; x, y)\right)\right] z_{i}\left(a_{k}, g_{i}[z](0 ; x, y)\right)\right| \\
& +\mid \sum_{k=1}^{r}\left[h_{k i}\left(0, g_{i}[\bar{z}](0 ; x, y)\right)\left[z_{i}\left(a_{k}, g_{i}[z](0 ; x, y)\right)-\bar{z}_{i}\left(a_{k}, g_{i}[\bar{z}](0 ; x, y)\right)\right] \mid\right. \\
& +\mid \int_{0}^{x} f_{i}\left(t, g_{i}[z](t ; x, y), z_{\left(t, g_{i}[z](t ; x, y)\right)}\right)-f_{i}\left(t, g_{i}[\bar{z}](t ; x, y), \bar{z}_{\left.\left.\left(t, g_{i}[\bar{z}](t ; x, y)\right)\right)\right] d t \mid}\right. \\
& \leq\left(R_{a} L_{a} K_{a}+L_{1 a}+\bar{p}\right)\|z-\bar{z}\|_{a}, \quad(x, y) \in I_{a},
\end{aligned}
$$


and

$$
\begin{gathered}
\left|(T z)_{i}(x, y)-(T \bar{z})_{i}(x, y)\right| \leq \mid \sum_{k=1}^{r} h_{k i}(x, y)\left[z_{i}\left(a_{k}+x, y\right)-\bar{z}_{i}\left(a_{k}+x, y\right) \mid\right. \\
\leq \bar{p}\|z-\bar{z}\|_{a}, \quad(x, y) \in I .
\end{gathered}
$$

Thus, it follows from (8) that $T$ is a contraction.

It remains to prove that the fixed point $z$ of the operator $T$ is the Carathéodory solution of (1), (2). By taking $y=g_{i}(x ; 0, \eta)$ in the equation $z_{i}(x, y)=(T z)_{i}(x, y)$, we get

$$
\begin{aligned}
z_{i}\left(x, g_{i}(x ; 0, \eta)\right)=\varphi_{i}(0, \eta) & -\sum_{k=1}^{r} h_{k i}(0, \eta) z_{i}\left(a_{k}, \eta\right) \\
& +\int_{0}^{x} f_{i}\left(t, g_{i}(t ; 0, \eta), z_{\left(t, g_{i}(t ; 0, \eta)\right)}\right) d t
\end{aligned}
$$

since the solution $g_{i}$ of (5) satisfies the following group property $g_{i}\left(t^{\prime} ; t, g_{i}(t ; x, y)\right)=g_{i}\left(t^{\prime} ; x, y\right)$. By differentiation of (11) with respect to $x$, using the chain rule differentiation Lemma (4.ii) of [7], and by putting again $y=g_{i}(x ; 0, \eta)$, we obtain that $z$ satisfies (1) almost everywhere in $I_{a}$. It follows immediately that $z$ satisfies (2). The proof of Theorem 1 is complete.

\section{Special cases of system (1)}

We list below a few examples of problems which can be derived from (1) by specializing the functions $\varrho$ and $f$.

1) Suppose that $\bar{\varrho}: I_{a_{0}} \times \mathbb{R}^{n} \times \mathbb{R}^{n} \rightarrow \mathbb{R}^{n m}$ and $\bar{f}: I_{a_{0}} \times \mathbb{R}^{n} \times \mathbb{R}^{n} \rightarrow \mathbb{R}^{n}$ are given functions. Let

$$
\begin{aligned}
& \varrho(x, y, v)=\bar{\varrho}\left(x, y, v(0,0), \int_{D} v(s, t) d s d t\right), \\
& f(x, y, v)=\bar{f}\left(x, y, v(0,0), \int_{D} v(s, t) d s d t\right), \quad(x, y, v) \in I_{a} \times C\left(D, \mathbb{R}^{n}\right) .
\end{aligned}
$$

Then system (1) reduces to the differential-integral system

$$
\begin{gathered}
D_{x} z_{i}(x, y)+\sum_{j=1}^{m} \bar{\varrho}_{i j}\left(x, y, z(x, y), \int_{D} z(x+s, y+t) d s d t\right) D_{y_{j}} z_{i}(x, y) \\
=\bar{f}_{i}\left(x, y, z(x, y), \int_{D} z(x+s, y+t) d s d t\right), \quad i=1, \ldots, n .
\end{gathered}
$$


2) Suppose that $\alpha=\left(\alpha_{0}, \alpha^{\prime}\right), \beta=\left(\beta_{0}, \beta^{\prime}\right): I_{a_{0}} \times C\left(D, \mathbb{R}^{n}\right) \rightarrow \mathbb{R}^{1+m}$, $\bar{\varrho}: I_{a_{0}} \times \mathbb{R}^{n} \times \mathbb{R}^{n} \rightarrow \mathbb{R}^{n m}$ and $\bar{f}: I_{a_{0}} \times \mathbb{R}^{n} \times \mathbb{R}^{n} \rightarrow \mathbb{R}^{n}$ are given. Assume that

$$
\begin{gathered}
\left(\alpha_{0}(x, y, v)-x, \alpha^{\prime}(x, y, v)-y\right) \in D \\
\left(\beta_{0}(x, y, v)-x, \beta^{\prime}(x, y, v)-y\right) \in D
\end{gathered}
$$

for $(x, y, v) \in I_{a_{0}} \times C\left(D, \mathbb{R}^{n}\right)$.

Put

$$
\begin{aligned}
& \varrho(x, y, v)=\bar{\varrho}\left(x, y, v(0,0), v\left(\alpha_{0}(x, y, v)-x, \alpha^{\prime}(x, y, v)-y\right)\right) \\
& f(x, y, v)=\bar{f}\left(x, y, v(0,0), v\left(\beta_{0}(x, y, v)-x, \beta^{\prime}(x, y, v)-y\right)\right) .
\end{aligned}
$$

Then system (1) reduces to the differential system with a retarded argument

$$
\begin{gathered}
D_{x} z_{i}(x, y)+\sum_{j=1}^{m} \bar{\varrho}_{i j}\left(x, y, z(x, y), z\left(\alpha_{0}\left(x, y, z_{(x, y)}\right), \alpha^{\prime}\left(x, y, z_{(x, y)}\right)\right)\right) D_{y_{j}} z_{i}(x, y) \\
=\bar{f}_{i}\left(x, y, z(x, y), z\left(\beta_{0}\left(x, y, z_{(x, y)}\right), \beta^{\prime}\left(x, y, z_{(x, y)}\right)\right)\right), \quad i=1, \ldots, n .
\end{gathered}
$$

The functions $\alpha$ and $\beta$ depend on the functional argument. Therefore, we cannot apply existence theorems from $[\mathbf{1 3}],[\mathbf{1 4}]$ to the above system.

3) If we take

$$
\begin{aligned}
& \varrho(x, y, v)=\bar{\varrho}\left(x, y, v(0,0),\left(V\left(\mathcal{I}_{(x, y)} v\right)\right)(x, y)\right), \\
& f(x, y, v)=\bar{f}\left(x, y, v(0,0),\left(V\left(\mathcal{I}_{(x, y)} v\right)\right)(x, y)\right),
\end{aligned}
$$

where $\left(\mathcal{I}_{(x, y)} v\right)(s, t)=v(s-x, t-y)$ then system (1) reduces to the system of differential-functional equations $([\mathbf{1 3}],[\mathbf{1 4}])$

$$
\begin{aligned}
D_{x} z_{i}(x, y)+\sum_{j=1}^{m} \bar{\varrho}_{i j}(x, y, z(x, y),(V z)(x, y)) D_{y_{j}} z_{i}(x, y) & \\
& =\bar{f}_{i}(x, y, z(x, y),(V z)(x, y)), \quad i=1, \ldots, n .
\end{aligned}
$$

\section{References}

1. P. Bassanini, The problem of Graffi-Cesari, Proc. Inter. Conf. Nonlinear Phenomena Math. Sci. Arlington, Acad. Press, USA (1982), 87-101. 
2. L. Byszewski, Existence and uniqueness of solutions of nonlocal problems for hyperbolic equation $u_{x t}=F\left(x, t, u, u_{x}\right)$, J. Appl. Math. Stochastic Anal. 3 (1990), 163-168.

3. L. Byszewski, Theorem about existence and uniqueness of continuous solution of nonlocal problem for nonlinear hyperbolic equation, Appl. Anal. 40 (1991), 173-180.

4. L. Byszewski, Existence of a solution of a Fourier nonlocal quasilinear parabolic problem, J. Appl. Math. Stochastic Anal. 5 (1992), $43-68$.

5. L. Byszewski, Monotone iterative method for a system of nonlocal initial-boundary parabolic problems, J. Math. Anal. Appl. 177 (1993), 445-458.

6. L. Byszewski and V. Lakshmikantham, Monotone iterative technique for nonlocal hyperbolic differential problem, J. Math. Phys. Sci. 26 (1992), 346-359.

7. L. Cesari, A boundary value problem for quasilinear hyperbolic system, Riv. Mat. Univ. Parma 3 (1974), 107-131.

8. J. Chabrowski, On non-local problems for parabolic equations, Nagoya Math. J. 93 (1984), 109-131.

9. T. CzŁAPIŃSKI, On existence and uniquenes of solutions of nonlocal problems for hyperbolic differential-functional equations, Preprint.

10. Z. Kamont And K. Topolski, Mixed problems for quasilinear hyperbolic differential-functional systems, Math. Balkanica 6 (1992), 313-324.

11. A. PLIś, Generalization of the Cauchy problem for a system of partial differential equations, Bull. Acad. Polon. Sci. Cl. III 4 (1956), $741-744$.

12. J. SzARski, Generalized Cauchy problem for differential-functional equations with first order partial derivatives, Bull. Acad. Polon. Sci. Ser. Sci. Math. Astr. Phys. 24 (1976), 575-580.

13. J. Turo, Local generalized solutions of mixed problems for quasilinear hyperbolic systems of functional partial differential equations in two independent variables, Ann. Polon. Math. 49 (1989), 259-278. 
14. J. Turo, Generalized solutions to functional partial differential equations of the first order,, Zeszyty Nauk. Politech. Gdańskiej Mat. 14 (1988), 1-99.

\author{
Department of Mathematics \\ Technical University of Gdańsk \\ 80-952 Gdańsk \\ Narutowicza 11/12 \\ POLAND \\ e-mail: turo@mif.pg.gda.pl
}

Primera versió rebuda el 30 de Maig de 1996, darrera versió rebuda el 17 de Març de 1997 